Jan-Hendrik Buhk

Kai Kallenberg

Alexander Mohr

Peter Dechent

Michael Knauth

\title{
Evaluation of angiographic computed tomography in the follow-up after endovascular treatment of cerebral aneurysms-a comparative study with DSA and TOF-MRA
}

Received: 7 April 2008

Revised: 3 August 2008

Accepted: 17 August 2008

Published online: 23 September 2008

(C) The Author(s) 2008. This article is published with open access at Springerlink. com

\author{
J.-H. Buhk $(\bowtie) \cdot$ K. Kallenberg · \\ A. Mohr · M. Knauth \\ Department of Neuroradiology, \\ University of Goettingen, \\ Robert-Koch-Str. 40, \\ 37075 Goettingen, Germany \\ e-mail: jh.buhk@mac.com \\ Tel.: +49-551-396643 \\ Fax: +49-551-3912868 \\ J.-H. Buhk · K. Kallenberg · P. Dechent \\ MR-Research in Neurology and \\ Psychiatry, University of Goettingen, \\ Robert-Koch-Str. 40, \\ 37075 Goettingen, Germany
}

\begin{abstract}
Following coil embolization of intracranial aneurysms, many centers perform at least one digital subtraction angiography (DSA) continuing with time-of-flight magnetic resonance angiography (TOF-MRA). Angiographic computed tomography (ACT) provides high-resolution data from a rotational acquisition of a c-arm-mounted flat panel detector. This study evaluates possible advantages of applying ACT in aneurysm follow-up. In 22 patients DSA examinations with a rotational acquisition were performed. Rotational data were processed into an isotropic highresolution volume. TOF-MRA was performed the day before DSA. Three experienced neuroradiologists performed a rating of the occlusion rate and a subjective method comparison. Weighted $\mathrm{k}$ statistics were calculated to assess the level of
\end{abstract}

interobserver agreement. Compared to DSA, the diagnostic value of ACT as well as of TOF-MRA was rated to be inferior, although the sensitivity of detecting residual necks was higher with both techniques. Compared to TOF-MRA, ACT achieves favorable ratings only in aneurysms after stentremodeling. Interobserver agreement was high for all techniques. Ratings of the occlusion rate correlated highly between all observers $(r>0.85$, $\mathrm{p}<0.001$, respectively). In selected patients ACT can add valuable diagnostic information to DSA. TOF-MRA remains a highly sensitive method for aneurysm follow-up.

Keywords Aneurysm - Coiling · Follow-up - Rotational angiography · Angiographic ct $\cdot$ Computed tomography $\cdot$ MR-angiography

\section{Introduction}

Endovascular treatment of cerebral aneurysms currently is a widely accepted therapeutic option in patients with acute subarachnoid hemorrhage (SAH) or unruptured aneurysms $[1,2]$. A long-term follow-up is mandatory because there is a relevant rate of aneurysm recurrence of up to $30 \%$, which does not always need retreatment, but, however, can be associated with a risk of rebleeding [3-6]. Many neuro- vascular centers perform a follow-up digital subtraction angiography (DSA) and a time-of-flight magnetic resonance angiography (TOF-MRA) at 3 to 6 months after treatment in order to continue with TOF-MRA alone, which has been shown to be sufficient in detecting recurrent aneurysms in the majority of the patients [7-9].

Three-dimensional rotational angiography (3D-RA) is an angiographic tool that has primarily been used to visualize cerebral aneurysms in a $3 \mathrm{D}$ surface-shaded or 
volume-rendered reconstruction in order to find the best therapeutic approach. Possible advantages of 3D-RA in follow-up examinations after endovascular treatment of cerebral aneurysms have not yet been described. The possibility of processing multiplanar reformatted slice images in high quality derived from a rotational dataset has been improved simultaneously to the development of the latest flat-panel detector-equipped angiographic devices. These systems provide a much higher acquisition speed and image information density combined with a large rectangular field of view. Rotational acquisitions by such detectors provide an almost CT-like contrast resolution allowing imaging of brain parenchyma, which can be of importance during neuro-interventional procedures [10, 11]. In addition, the spatial resolution is higher than in current helical CT: Angiographic CT (ACT) - generated by rotational acquisition - provides an isotropic resolution of about $0.1 \mathrm{~mm}$ in comparison to about $0.5-\mathrm{mm}$ maximum $\mathrm{CT}$ resolution [12]. The value of ACT in peri-interventional imaging of intracranial stents has been shown, especially the combination of 3D volume-rendered (VRT) and thinand thick-sliced maximum intensity projected (MIP) reconstructions provides additional information to conventional DSA [13, 14].

The purpose of the representative cross-sectional study presented here is to systematically elucidate possible diagnostic advantages of ACT compared to DSA and TOF-MRA in follow-up examinations of patients after endovascular treatment of cerebral aneurysms.

\section{Methods}

\section{Patients}

Twenty-two patients treated by endovascular coil embolization of a cerebral aneurysm in our department (14 men, median age 48 years, range $29-78$ years) participated in the prospective study. All patients were admitted for standard 6-month follow-up DSA, which included a rotational acquisition. TOF-MRA was performed the day before the DSA examination. Aneurysm locations were the following: anterior communicating artery (Acom): $n=8$; internal carotid artery (ICA): $\mathrm{n}=5$; posterior communicating artery (Pcom): $n=4$; basilar artery (BA): $n=4$; posterior inferior cerebellar artery (PICA): $n=1$. Stent-remodeling was performed in seven patients $\left(6 \mathrm{x}\right.$ Neuroform ${ }^{\mathrm{TM}}$, Boston Scientific, Natick, MA; 1 x Driver ${ }^{\mathrm{TM}}$, Medtronic Inc., Minneapolis, MN). Approval of the local ethics committee and informed patient consent were obtained.

\section{Image acquisition}

Rotational acquisitions and conventional DSA examinations were performed on an AxiomArtis dBA biplane angiography system equipped with flat panel detectors (Siemens Medical Solutions, Forchheim, Germany). The rotational acquisitions were performed with the following parameters, complying with the standard 3D-RA protocol: dual 10-s rotation (mask images first, then images during intra-arterial contrast media injection), 273 projections each, $30 \mathrm{~cm} \times 40 \mathrm{~cm}$ detector size, radiation dose: $0.36 \mu \mathrm{Gy}$ per image, $\mathrm{CTDI}_{\text {weighted }} \approx 22 \mathrm{mGy}$ (manufacturer information).

Diagnostic magnetic resonance imaging (MRI) and TOF-MRA scans were performed at $3 \mathrm{~T}$ (Magnetom Trio, Siemens Medical Solutions, Erlangen, Germany). Parameters of the TOF-MRA were the following: repetition time: $28 \mathrm{~ms}$, echo time: $4.6 \mathrm{~ms}$, matrix size: $448 \times 448$, $0.6 \mathrm{~mm}$ slice thickness, acquisition time: 5:12 min.

\section{Image postprocessing}

DSA series were processed by standard optimization of the mask images and pixel shifting. Postprocessing of the rotational image data into a volume data set (ACT) was performed using dedicated commercial software on a medical workstation ("Leonardo," DynaCTTM, InSpace $3 \mathrm{D}^{\mathrm{TM}}$, Siemens Medical Solutions, Forchheim, Germany). The software includes the application of system-specific filter algorithms in order to correct for beam hardening, scattered radiation, truncated projections and ring artifacts. Postprocessing resulted in isotropic volume data sets defined by a batch of 300-400 slices in a $512 \times 512$ matrix. Isotropic resolution was in the order of $0.1 \mathrm{~mm}$. For diagnostic evaluation the ACT data were viewed as multiplanar MIP slices in free orientations as well as 3D MIP and 3D VRT images. TOF-MRA data were processed according to the ACT data. DSA data only included twodimensional series. All image viewing was performed on a Macintosh workstation (Apple Inc., Cupertino, CA) with OsiriX ${ }^{\mathrm{TM}}$ installed [15].

\section{Statistical analysis}

Three experienced neuroradiologists (K.K., A.M., M.K.) independently performed the image viewing and rating. Firstly, the state of aneurysm occlusion was rated following a three-step ordinal scale ("complete occlusion" vs. "residual neck" vs. "residual aneurysm") [6]. Pearson's correlation coefficient was calculated for each pair of methods in the diagnostic rating. Weighted K-statistics were calculated in order to assess interobserver variabilities; a value above $\mathrm{k}=0.6$ was assumed to represent substantial agreement $[16,17]$. Secondly, a direct methodcomparison questionnaire in order to achieve a statement of diagnostic superiority vs. equality vs. inferiority was completed by each observer. A second questionnaire regarding TOF-MRA vs. ACT was completed concerning the artifact load of the technique. 
Table 1 Cumulative results of the aneurysm occlusion rate by three observers

\begin{tabular}{llll}
\hline Occlusion & DSA & TOF-MRA & ACT \\
\hline Complete occlusion & 38 & 34 & 36 \\
Residual neck & 12 & 16 & 14 \\
Residual aneurysm & 16 & 13 & 16 \\
Overall & 66 & 63 & 66 \\
\hline
\end{tabular}

One patient with residual aneurysm was excluded from the TOFMRA rating as non-applicable because of clip-related artifacts (see also Fig. 1)

\section{Results}

\section{Occlusion rate}

The cumulative results of the ratings of occlusion rate are shown in Table 1. All residual aneurysms were consistently detected with every technique except in one patient, where TOF-MRA was impaired due to clip-related signal extinction (Fig. 1a-d). That patient was excluded from the TOFMRA rating.

Correlation analysis (Pearson) of occlusion rate revealed the following results: DSA vs. TOF-MRA: $r=0.889(\mathrm{P}<$ $0.001)$; DSA vs. ACT: $r=0.893(\mathrm{P}<0.001)$; ACT vs. TOF-
MRA: $r=0.913(\mathrm{P}<0.001)$. TOF-MRA achieved the highest score by depicting 16 residual necks, followed by ACT (14) and DSA (12).

The case presented in Fig. 2 illustrates a residual neck of an Acom aneurysm, which is equally well detectable in ACT as well as in TOF-MRA (Fig. 2c,d), but not in DSA due to the unfavorable orientation (Fig. 2a,b). The residual aneurysm presented in Fig. 3 is reliably detected in all three techniques (Fig. 3a,b: DSA; c: TOF-MRA; d: ACT). The stretched and partially endothelialized coil remnant in the right ICA and A1 is well visualized by ACT (Fig. 3d).

Table 2 presents the results of the $K$ statistics. Interobserver agreement was very good for all groups $\left(\kappa_{\mathrm{w}}>0.8\right)[16,17]$.

\section{Direct comparison}

All data are presented cumulatively. Firstly, a subjective assessment of the diagnostic performance of each technique compared to the other was made. Both ACT and TOF-MRA received inferior ratings when compared to DSA, being the gold standard (MRA vs. DSA: favors MRA: $n=14$, no preference: $n=16$, favors DSA: $n=33$; ACT vs. DSA: favors ACT: $n=14$, no preference: $n=15$; favors DSA: $n=37$ ).
Fig. 1 Follow-up examination of a 51-year-old female patient with history of subarachnoid hemorrhage (SAH) and neurosurgical as well as interventional therapy of a recurrent ICA aneurysm. The DSA series in optimized projection with selective injection into right ICA (a: unsubtracted, b: subtracted) reveals an aneurysm recurrence beyond the coil package. On the opposite of the recurrent aneurysm there is an infundibular origin of the posterior communicating artery (Pcom). TOFMRA is not diagnostic in this case due to clip-related artifacts (c). A thin-sliced coronal reconstructed ACT (d: $5 \mathrm{~mm}$ MIP) demonstrates the refilling inside the coil package (d, arrows). Endovascular re-treatment was performed a few days later
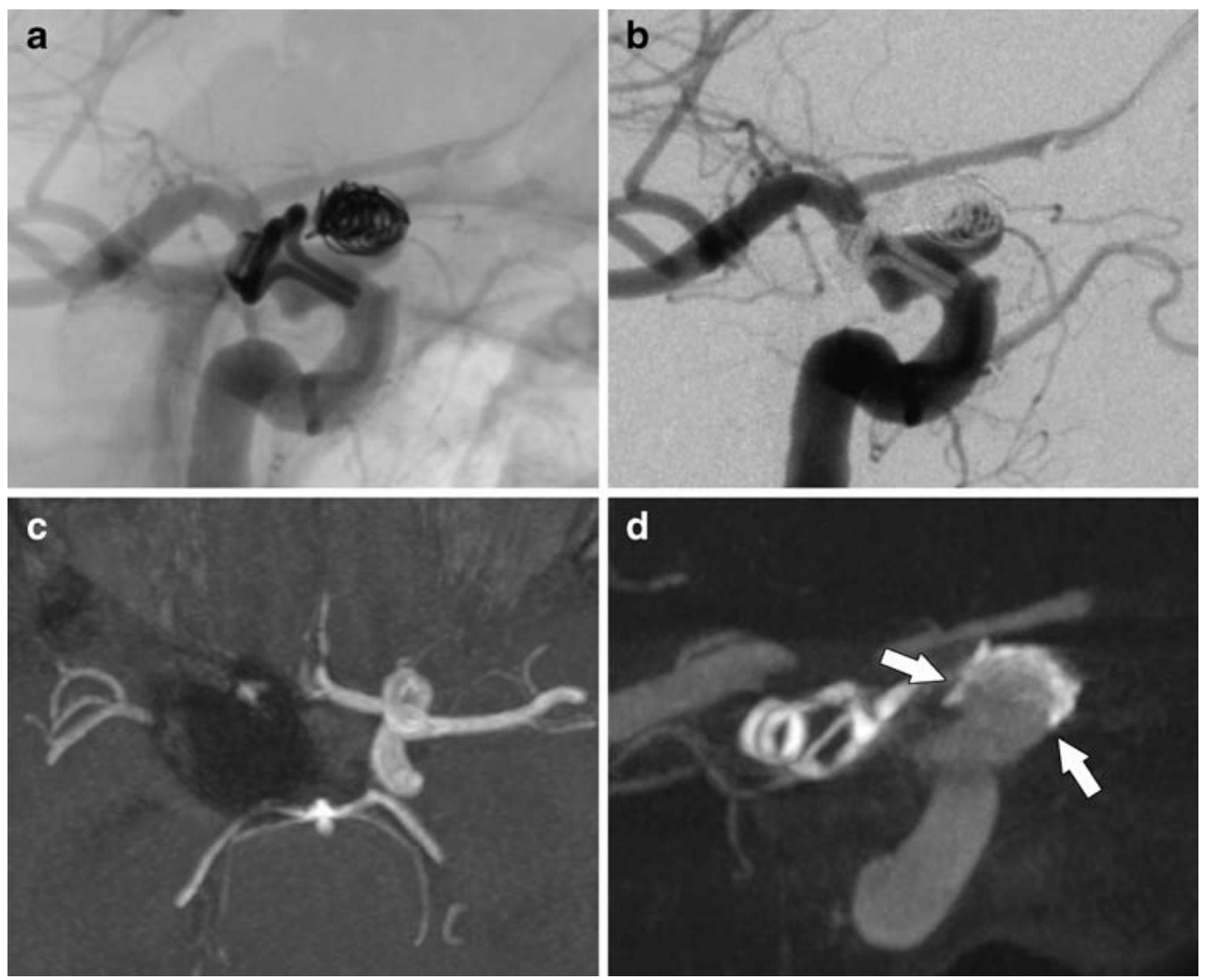
Fig. 2 Follow-up examination of a 59-year-old male patient with history of SAH and interventional therapy of an anterior communicating artery (Acom) aneurysm. The DSA series in anterior projection (a: unsubtracted, b: subtracted) seems to show a complete occlusion and was rated like that by all observers. Both thin-sliced ACT (c: 2-mm MIP, transversal) and TOF-MRA (d: 10-mm MIP, coronal) revealed a small neck remnant (c, d, arrows)
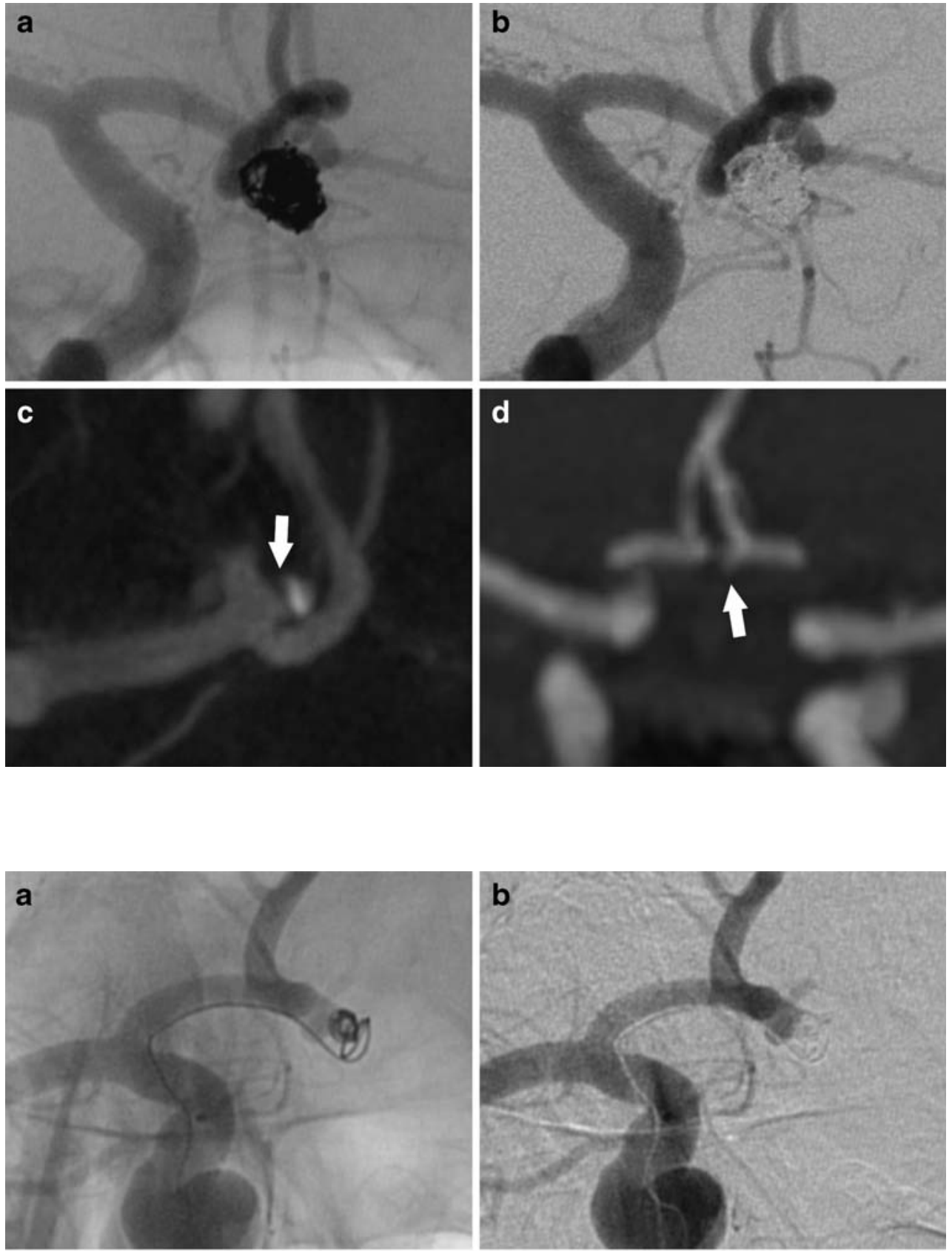
Fig. 3 Follow-up examination
of 61-year-old male patient with history of SAH and interventional therapy of an Acom aneurysm complicated by coil stretching. The DSA series in anterior orientation (a: unsubtracted, b: subtracted) shows a large basal refilling. TOF-MRA reveals a corresponding finding (c: $10-\mathrm{mm}$ MIP, arrow). Thinsliced reconstructions of ACT data show the basal refilling (d, closed arrow) as well as a slim rim of attenuation close to the stretched coil in the A1 segment that can be interpreted as neointima (d, open arrow)
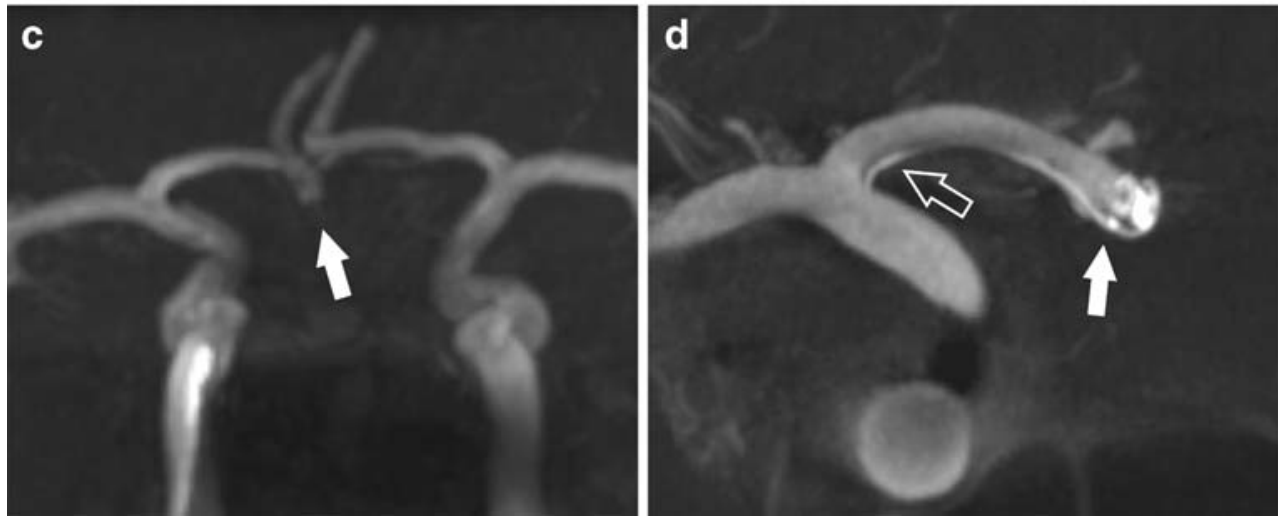
Table 2 Interobserver agreement regarding the occlusion rate, measured as $\mathrm{kw}$, achieves substantially high agreement for all techniques

\begin{tabular}{llll}
\hline & DSA & TOF-MRA & ACT \\
\hline Observers 1 vs. 2 & 0.81 & 0.78 & 0.72 \\
Observers 1 vs. 3 & 0.78 & 0.78 & 0.81 \\
Observers 2 vs. 3 & 0.87 & 0.84 & 0.81 \\
Mean $\mathrm{K}_{\mathrm{w}}$ & 0.82 & 0.80 & 0.78 \\
\hline
\end{tabular}

Comparing MRA to ACT presents a slight trend in favor of TOF-MRA (favors MRA: $n=29$; no prevalence: $n=14$; favors ACT: $n=20)$. Dividing these data into two subgroups (aneurysms that have been treated with stent remodeling, $n=21$ vs. those that have been treated without stent remodeling, $\mathrm{n}=42$ ) reveals a trend towards TOFMRA being rated superior to ACT when no stent had been implanted, while the methods seem to be equivalent in the group of patients with stent remodeling.

Secondly, a subjective assessment of the impairments due to artifact load was asked in comparison between TOFMRA and ACT. We were aware of not defining the character of artifacts we asked for because many ACT artifacts have not been described yet and are therefore hard to anticipate. Comparable to the ratings of the diagnostic value, TOF-MRA is superior to ACT when no stent was implanted (favors MRA: $n=23$, no prevalence: $n=7$, favors
ACT: $n=12$ ). A slight superiority of ACT is observed for patients with stent remodeling (favors MRA: $n=7$, no prevalence: $n=3$, favors ACT: $n=11$ ). A second subgroup analysis was performed regarding the aneurysm size. A small aneurysm was defined as having a maximal diameter below $10 \mathrm{~mm}(\mathrm{n}=13$ patients), a large aneurysm having a maximal diameter of $10 \mathrm{~mm}$ or more ( $\mathrm{n}=8$ patients). Here equivalence results when comparing the artifact load in TOF-MRA and ACT regarding small aneurysms (favors MRA: $n=14$, no prevalence: $n=8$, favors ACT: $n=17$ ), but a clear superiority of TOF-MRA over ACT regarding large aneurysms (favors MRA: $n=16$, no prevalence: $n=2$, favors ACT: $n=6$ ).

One crucial artifact of ACT is demonstrated in Fig. 4. A large ophthalmoplegic ICA aneurysm was treated by stentprotected coil embolization. The follow-up DSA showed a slight central residual filling. ACT provides good visual-
Fig. 4 Follow-up examination of a 63-year-old female after endovascular therapy of an ophthalmoplegic left ICA aneurysm. The subtracted DSA series in optimized projection (a) show hints for a minimal central residual filling (arrow). A thicksliced MIP of the ACT data (b) is helpful in displaying the configuration of the coil package and the relation to the ophthalmic artery, but does not reveal the central filling. A thinsliced section in the same orientation (c, 2-mm MIP) shows an amorphous structure of the core of the coil package (arrow) not delineating any residual filling. The impression of this artifact is similar to an eggshell; therefore, we chose the denomination "eggshell artifact." TOF-MRA succeeds in delineating the central filling when a thin-sliced MIP section is processed (d, arrow)
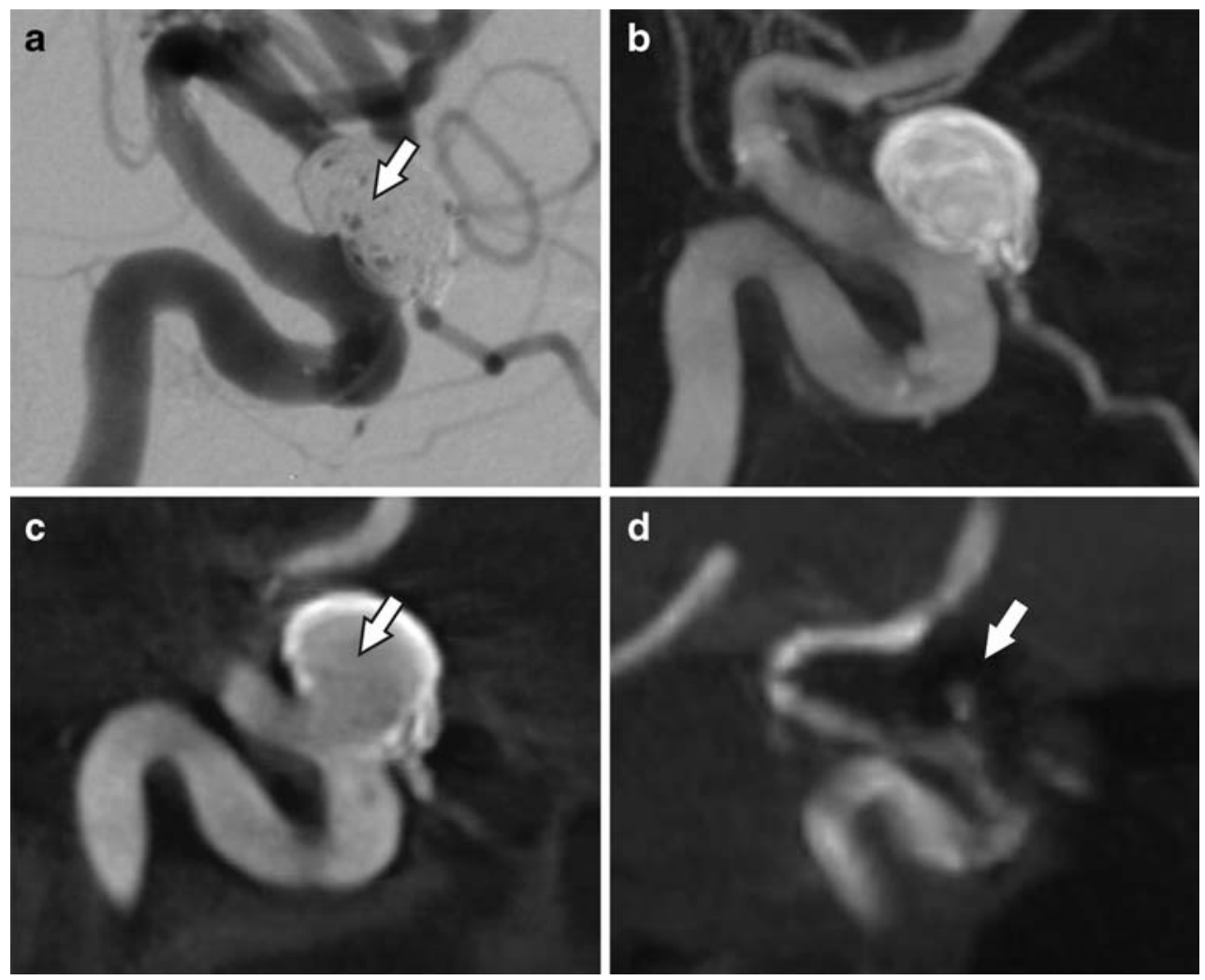
ization of the large coil package using 3D MIP. Concerning the interior of the coil package, ACT suffers from hardening artifacts presenting as an amorphous attenuation comparable to the contrast media filling of the parent vessel. Performing a subtraction only shows some movement artifacts on the edge of the coil package, but no residual filling. The residual filling is confirmed by TOF-MRA.

\section{Discussion}

We are aware that many neuroendovascular centers perform rotational acquisitions as a component of a follow-up angiographic examination. For the purpose of this study we separated the one examination into the two independent techniques.

In our study DSA alone achieved good results in direct comparison to ACT as well as to TOF-MRA. The striking advantage is of course the dynamic information that is missing in both ACT and conventional TOF-MRA. Therefore, we believe that currently no less invasive angiographic technique can completely replace DSA for follow-up after endovascular therapy of cerebral aneurysms. The purpose of this study was therefore to evaluate whether there is a group of patients that should additionally be examined with advanced techniques like ACT.

The scoring of the occlusion rate yielded highly consistent results for all techniques and for all three observers. Therefore, ACT can, in principle, be assumed to be a reliable technique in diagnostic imaging of cerebral aneurysms after coil embolization. The results of our study also underline the high value of TOF-MRA in aneurysm follow-up. Our study confirms earlier results of the very high sensitivity for residual flow in cerebral aneurysms treated with coil embolization [8, 9, 18-20].

We experienced some TOF-MRA artifacts mainly related to the susceptibility artifacts caused by coils and stents. Especially stent-related artifacts impair the assessment of the lumen of the parent vessel. The possibility of almost artifact-free imaging of intracranial stents by ACT has been described before $[13,21]$. Although our data do not show a convincing superiority, ACT may have some advantages in patients after stent remodeling. However, these are often patients with large aneurysms. The algorithms for reconstructing rotational radiographic data have been found to be insufficient for the assessment of large coil packages resulting in an amorphous signal in the interior of the coil packages ("eggshell" artifact). Similar results in applying ACT to monitor stent and coil apposition during neurointerventional procedures have been published recently [14]. Both conventional 3D-RA and ACT did not consistently detect residual fillings in larger aneurysms. An angiographic follow-up examination can therefore be done without a rotational acquisition, as proposed before [9].

In patients after treatment of complex aneurysms, there seem to be advantages of the 3D techniques over plain DSA (see Figs. 1, 2). Neurosurgical clips disqualify TOFMRA as a follow-up technique (see Fig. 1); in such patients ACT can provide valuable information.

\section{Conclusion}

ACT is a diagnostic tool that has useful added value to conventional DSA in treated aneurysms with a complex anatomy. It may have advantages over TOF-MRA in aneurysms treated with stent remodeling. Larger coil packages (approximately over $10-\mathrm{mm}$ diameter) produce substantial artifacts in ACT data; therefore, we would advise not to use ACT, but rather MRA in such patients.

Disclosure The authors declare that there is no conflict of interest.

Open Access This article is distributed under the terms of the Creative Commons Attribution Noncommercial License which permits any noncommercial use, distribution, and reproduction in any medium, provided the original author(s) and source are credited.

\section{References}

1. Molyneux A, Kerr R, Stratton I et al (2002) International Subarachnoid Aneurysm Trial (ISAT) of neurosurgical clipping versus endovascular coiling in 2143 patients with ruptured intracranial aneurysms: a randomised trial. Lancet 360:1267-1274

2. Wiebers DO, Whisnant JP, Huston J et al (2003) Unruptured intracranial aneurysms: natural history, clinical outcome, and risks of surgical and endovascular treatment. Lancet 362:103-110
3. Campi A, Ramzi N, Molyneux AJ et al (2007) Retreatment of ruptured cerebral aneurysms in patients randomized by coiling or clipping in the International Subarachnoid Aneurysm Trial (ISAT). Stroke 38:1538-1544

4. Li MH, Gao BL, Fang C et al (2006) Angiographic follow-up of cerebral aneurysms treated with Guglielmi detachable coils: an analysis of 162 cases with 173 aneurysms. AJNR Am J Neuroradiol 27:1107-1112
5. Piotin M, Spelle L, Mounayer C et al (2007) Intracranial aneurysms: treatment with bare platinum coils-aneurysm packing, complex coils, and angiographic recurrence. Radiology 243:500-508

6. Raymond J, Guilbert F, Weill A et al (2003) Long-term angiographic recurrences after selective endovascular treatment of aneurysms with detachable coils. Stroke 34:1398-1403 
7. Cottier JP, Bleuzen-Couthon A, Gallas S et al (2003) Follow-up of intracranial aneurysms treated with detachable coils: comparison of plain radiographs, 3D time-of-flight MRA and digital subtraction angiography. Neuroradiology 45:818-824

8. Okahara M, Kiyosue H, Hori Y et al (2004) Three-dimensional time-offlight MR angiography for evaluation of intracranial aneurysms after endosaccular packing with Guglielmi detachable coils: comparison with 3D digital subtraction angiography. Eur Radiol 14:1162-1168

9. Yamada N, Hayashi K, Murao K et al (2004) Time-of-flight MR angiography targeted to coiled intracranial aneurysms is more sensitive to residual flow than is digital subtraction angiography. AJNR Am J Neuroradiol 25:1154-1157

10. Engelhorn T, Struffert T, Richter G et al (2008) Flat panel detector angiographic $\mathrm{CT}$ in the management of aneurysmal rupture during coil embolization. AJNR Am J Neuroradiol doi:10.3174/ajnr. A1119
11. Heran NS, Song JK, Namba K et al (2006) The utility of DynaCT in neuroendovascular procedures.

AJNR Am J Neuroradiol 27:330-332

12. Kalender WA (2003) The use of flatpanel detectors for CT imaging. Radiologe 43:379-387

13. Benndorf G, Strother CM, Claus B et al (2005) Angiographic CT in cerebrovascular stenting. AJNR Am J Neuroradiol 26:1813-1818

14. Richter G, Engelhorn T, Struffert T et al (2007) Flat panel detector angiographic CT for stent-assisted coil embolization of broad-based cerebral aneurysms. AJNR Am J Neuroradiol 28:19021908

15. Rosset A, Spadola L, Ratib O (2004) OsiriX: an open-source software for navigating in multidimensional DICOM images. J Digit Imaging $17: 205-216$

16. Cohen J (1968) Weighted kappa: Nominal scale agreement with provision for scaled disagreement or partial credit. Psychol Bull 70:213-220

17. Landis JR, Koch GG (1977) The measurement of observer agreement for categorical data. Biometrics 33:159 174
18. Deutschmann HA, Augustin M, Simbrunner J et al (2007) Diagnostic accuracy of 3D time-of-flight MR angiography compared with digital subtraction angiography for follow-up of coiled intracranial aneurysms: influence of aneurysm size. AJNR Am J Neuroradiol 28:628-634

19. Majoie CB, Sprengers ME, van Rooij WJ et al (2005) MR angiography at $3 \mathrm{~T}$ versus digital subtraction angiography in the follow-up of intracranial aneurysms treated with detachable coils. AJNR Am J Neuroradiol 26:13491356

20. Westerlaan HE, van der Vliet AM, Hew JM et al (2005) Time-of-flight magnetic resonance angiography in the follow-up of intracranial aneurysms treated with Guglielmi detachable coils. Neuroradiology 47:622-629

21. Buhk JH, Lingor P, Knauth M (2008) Angiographic CT with intravenous administration of contrast medium is a noninvasive option for follow-up after intracranial stenting. Neuroradiology 50:349-354 